Research Article

\title{
Low-Complexity Decentralized Active Damping of One-Dimensional Structures
}

\author{
Petr Hušek (D), ${ }^{1}$ Filip Svoboda, ${ }^{1}$ Martin Hromčík, ${ }^{1}$ and Zbyněk Šika ${ }^{2}$ \\ ${ }^{1}$ Department of Control Engineering, Faculty of Electrical Engineering, Czech Technical University in Prague, Technická 2, \\ 16627 Prague, Czech Republic \\ ${ }^{2}$ Department of Mechanics, Biomechanics and Mechatronics, Faculty of Mechanical Engineering, \\ Czech Technical University in Prague, Technická 4, 16627 Prague 6, Czech Republic \\ Correspondence should be addressed to Petr Hušek; husek@fel.cvut.cz
}

Received 22 August 2018; Revised 12 October 2018; Accepted 28 October 2018; Published 2 December 2018

Academic Editor: Tai Thai

Copyright (๑ 2018 Petr Hušek et al. This is an open access article distributed under the Creative Commons Attribution License, which permits unrestricted use, distribution, and reproduction in any medium, provided the original work is properly cited.

In the paper, we propose distributed feedback control laws for active damping of one-dimensional mechanical structures equipped with dense arrays of force actuators and position and velocity sensors. We consider proportional position and velocity feedback from the neighboring nodes with symmetric gains. Achievable control performance with respect to stability margin and damping ratio is discussed. Compared to full-featured complex controllers obtained by modern design methods like LQG, H-infinity, or mu-synthesis, these simplistic controllers are more suitable for experimental fine tuning and are less case-dependent, and they shall be easier to implement on the target future smart-material platforms.

\section{Introduction}

The established paradigm in past and current active damping projects is as follows: the mechanical object is defined first (plate, beam, car door, wing panel, etc.). Systems detailed design and modelling phases follow the methods in $[1,2]$ giving rise to very accurate FEM models with tens of thousands of degrees of freedom. Alternatively for existing prototypes, the experimental identification approach can be applied to get the mathematical models directly via experimental modal analysis [3]. Model order reduction [1, 4] then gives accurate enough yet tractable models for optimal actuators and sensors placement [5-7]. Finally, a very limited number of them are considered (say up to twenty) for the design of the control laws $[1,8]$. Finally, validation and verification of the solutions by high-fidelity simulations is performed, followed by laboratory experiments and final deployment of the product. For any new project-or even a relatively mild modification of a previously accomplished project-all these steps must be performed (or re-visited) again. Implications towards requested research and development costs are significant and obvious.
Therefore, there is a need to use other type of control methodologies, and recent advances in MEMS sensors and microactuators, ongoing intensive research on new smart materials, and progress in computational power pave the way to massive development of heavily distributed control in this context.

Distributed control is now a very active field of research, thanks to potential applications which require high scalability and reliability. The main advantage of using distributed control is the locality of the necessary measurement and actuation-the measurements are collected and processed in a distributed manner. This kind of control can be applied for automated highway systems [9], car formations [10], and also flexible structures. The work in [11], for instance, studies a flexible beam model with bending and torsion motions, and a distributed arrangement with two force-actuators and three moment-actuators paired with rate gyros was elaborated. In [12], a dense network of piezoelectric patch actuators was proposed to realize the distributed actuation. In [13], a distributed piezoelectric actuation was involved and applied to the placement problem of patches so that the deformations are suppressed 
at preselected locations. Multipositive feedback approach for flexible structure control was presented in [14]. Since the flexible systems are passive by nature, one can also employ a lot of results available for distributed control of the passive system $[15,16]$. Completely passive solutions can be obtained using piezostructures, as reported in [17].

One of the natural goals when dealing with control of flexible mechanical structures is vibration suppression. One standard approach relies on application of a large number of neutralizers placed in prespecified locations along the structure composed from masses and springs. The goal is not only to design the neutralizers' parameters but specify their locations as well since vibrations can be eliminated only at the attachment point of the vibrating beam while amplification of vibration may occur in other parts of the beam. Dynamic vibration absorbers using magnetorheological elastomers were used in [18]. In [19, 20], a set of optimum conditions for global control of the kinetic energy based on the fixed-points theory was proposed. Dynamic transfer matrices using mobility or impedance were used in [21]. In [22], an iterative procedure was developed to find the required resonance frequencies of variable stiffness neutralizers to create nodes at selected locations. Wide-band frequency passive vibration attenuation design for the absorbers was introduced in [23]. In [24], explicit model predictive vibration control was tested. A different approach consists in control and attenuation of multiple travelling waves propagated in a one-dimensional structure [25-28]. Sliding mode control on seat vibration reduction problem was applied in [29].

\section{Structured Control Laws for Smart Materials}

The paper presents an attempt to systematic proportional decentralized position-velocity feedback for active damping of mechanical structures equipped with dense arrays of force actuators and position and velocity sensors. Such a control law is characterized by a very small number of parameters and simple procedures for their tuning compared to centralized approach. Although the results are presented for a one-dimensional structure model only, it is believed that a generalization to two-dimensional mechanical structures will be possible.

The research is motivated by vehicular platoon control where relative position and relative or absolute velocity feedback related to the preceding and succeeding vehicle is often considered [30-32]. Nevertheless, the measure of control performance in both applications is different. For vehicular platooning, the main goal consists in preserving a prescribed spacing between the vehicles and in keeping the leader's velocity, whereas when dealing with mechanical structures, a fast and adequate damping of the oscillating modes is required. Hence, the presented control design is focused on investigation of feasible damping ratio of the least damped mode and achievable stability margin of all modes.

Throughout the paper, the superscript $\mathrm{T}$ denotes transpose, $I_{n}$ stands for $n \times n$ identity matrix, $\operatorname{Re}(\cdot)$ and $\operatorname{Im}(\cdot)$ denotes real and imaginary part, respectively, $\otimes$ denotes the Kronecker product, and $\sigma(\cdot)$ denotes the spectrum of a matrix.

\section{One-Dimensional Structure Longitudinal Model}

Let us consider a one-dimensional structure composed from the masses $m$, springs $\bar{k}$, and dampings $\bar{b}$, each of the same value. Let us assume that the input forces may act on each individual mass independently and we are able to measure positions and velocities of each mass, i.e., actuators and sensors are placed in the same positions. Longitudinal vibrations of such a structure can be described by a state-space model:

$$
\dot{\bar{x}}=\bar{A} \bar{x}+\bar{B} \bar{u},
$$

where

$$
\bar{x}=\left[p_{1}, \dot{p}_{1}, p_{2}, \dot{p}_{2}, \ldots, p_{\eta}, \dot{p}_{\eta}\right]^{\mathrm{T}} \in \mathfrak{R}^{2 \eta},
$$

where $p_{i}, i=1, \ldots, \eta$, are the positions of the masses, $\eta$ is the number of nodes, and $\bar{u} \in \mathfrak{R}^{\eta}$ is the vector of the input forces [32].

The matrices $\bar{A} \in \mathfrak{R}^{2 \eta \times 2 \eta}$ and $\bar{B} \in \mathfrak{R}^{2 \eta \times \eta}$ are given by

$$
\begin{aligned}
& \bar{A}=I_{\eta} \otimes A_{1}+L \otimes A_{2}, \\
& \bar{B}=I_{\eta} \otimes\left(\left[\begin{array}{ll}
0 & 1
\end{array}\right]^{\mathrm{T}}\right),
\end{aligned}
$$

with

$$
\begin{aligned}
& A_{1}=\left[\begin{array}{ll}
0 & 1 \\
0 & 0
\end{array}\right], \\
& A_{2}=\left[\begin{array}{rr}
0 & 0 \\
-k_{0} & -b_{0}
\end{array}\right],
\end{aligned}
$$

where $k_{0}=\bar{k} / m, b_{0}=\bar{b} / m$. The matrix $L \in \mathfrak{R}^{\eta \times \eta}$ is the Laplacian of the graph corresponding to the structure which is in this case given as

$$
L=\left[\begin{array}{ccccc}
1 & -1 & & & \\
-1 & 2 & -1 & & \\
& \ddots & \ddots & \ddots & \\
& & -1 & 2 & -1 \\
& & & -1 & 1
\end{array}\right] .
$$

Since for practical reasons at least one of the nodes has to be fixed, the equation (1) becomes

$$
\dot{x}=A x+B u,
$$

where $x \in \mathfrak{R}^{2 n}, u \in \mathfrak{R}^{n}$ comes from $\bar{x}$ and $\bar{u}$ by omitting the entries corresponding to the fixed nodes, and $n$ is the number of the nonfixed nodes. by

The matrices $A \in \mathfrak{R}^{2 n \times 2 n}$ and $B \in \mathfrak{R}^{2 n \times n}$ are then given

$$
\begin{aligned}
& A=I_{n} \otimes A_{1}+L_{\mathrm{g}} \otimes A_{2}, \\
& B=I_{n} \otimes\left(\left[\begin{array}{ll}
0 & 1
\end{array}\right]^{\mathrm{T}}\right),
\end{aligned}
$$

where the matrix $L_{\mathrm{g}} \in \mathfrak{R}^{n \times n}$ is called the grounded Laplacian that results from Laplacian $L$ (5) by omitting the rows and 
columns corresponding to the fixed nodes. In the sequel, we will assume without loss of generality that the fixed nodes are the first and last one, $n=\eta-2$, and

$$
L_{\mathrm{g}}=\left[\begin{array}{ccccc}
2 & -1 & & & \\
-1 & 2 & -1 & & \\
& \ddots & \ddots & \ddots & \\
& & -1 & 2 & -1 \\
& & & -1 & 2
\end{array}\right] .
$$

The eigenvalues of $L_{\mathrm{g}}$ are all positive and are given by

$$
\lambda_{\ell}=2-2 \cos \left(\frac{\ell \pi}{n+1}\right), \quad \ell=1, \ldots, n .
$$

We will see later that from the eigenvalues (9), the minimum and maximum ones are of special interest. Those can be determined as

$$
\begin{aligned}
& \lambda_{\text {min }}=\min _{\ell} \lambda_{\ell}=\lambda_{1}=2-2 \cos \left(\frac{\pi}{n+1}\right), \\
& \lambda_{\text {max }}=\max _{\ell} \lambda_{\ell}=\lambda_{n}=2-2 \cos \left(\frac{n \pi}{n+1}\right) .
\end{aligned}
$$

In this paper, we will use distributed control law where the control action applied to each node depends symmetrically on relative positions and velocities with respect to its neighbors, i.e.,

$$
\begin{aligned}
u_{i}= & -k\left(p_{i+1}-p_{i}\right)-k\left(p_{i+1}-p_{i+2}\right)-b\left(\dot{p}_{i+1}-\dot{p}_{i}\right) \\
& -b\left(\dot{p}_{i+1}-\dot{p}_{i+2}\right), \quad i=1, \ldots, n, k, b \geq 0,
\end{aligned}
$$

with $p_{1}$ and $p_{n+2}$ being fixed (Figure 1).

The control law (11) can be written as

$$
u=K x=\left(L_{\mathrm{g}} \otimes\left[\begin{array}{ll}
k & b
\end{array}\right]\right) x .
$$

After substituting (12) into (6), one obtains the description of the closed-loop system:

$$
\dot{x}=A_{\mathrm{c}} x=(A+B K) x,
$$

where

$$
\begin{aligned}
A+B K & =I_{n} \otimes A_{1}+L_{\mathrm{g}} \otimes A_{2}+L_{\mathrm{g}} \otimes K_{2} \\
& =I_{n} \otimes A_{1}+L_{\mathrm{g}} \otimes\left(A_{2}+K_{2}\right),
\end{aligned}
$$

with

$$
K_{2}=\left[\begin{array}{cc}
0 & 0 \\
-k & -b
\end{array}\right]
$$

For determination of the eigenvalues of matrix $A_{c}$, we will use the following lemma.

Lemma 1 [32].

$$
\sigma\left(I_{n} \otimes \widetilde{A}_{1}+\widetilde{L} \otimes \widetilde{A}_{2}\right)=\bigcup_{\lambda_{\ell} \in \sigma(\widetilde{L})}\left\{\sigma\left(\widetilde{A}_{1}+\lambda_{l} \widetilde{A}_{2}\right)\right\}
$$
result:

$$
\begin{aligned}
\sigma\left(A_{\mathrm{c}}\right) & =\sigma\left(I_{n} \otimes A_{1}+L_{\mathrm{g}} \otimes\left(A_{2}+K_{2}\right)\right) \\
& =\bigcup_{\lambda_{\ell} \in \sigma\left(L_{\mathrm{g}}\right)}\left\{\sigma\left[\begin{array}{cc}
0 & 1 \\
-\lambda_{\ell}\left(k_{0}+k\right) & -\lambda_{\ell}\left(b_{0}+b\right)
\end{array}\right]\right\} .
\end{aligned}
$$

It turns out that the closed-loop eigenvalues are given as the roots of the characteristic equation:

$$
s^{2}+\lambda_{\ell}\left(b_{0}+b\right) s+\lambda_{\ell}\left(k_{0}+k\right)=0, \quad \ell=1, \ldots, n,
$$

i.e.,

$$
s_{\ell}^{ \pm}=\frac{-\lambda_{\ell}\left(b_{0}+b\right) \pm \sqrt{\lambda_{\ell}^{2}\left(b_{0}+b\right)^{2}-4 \lambda_{\ell}\left(k_{0}+k\right)}}{2}, \quad \ell=1, \ldots, n .
$$

\section{Control Strategies}

There are many options where to place the closed-loop eigenvalues. Nevertheless, from the vibration suppression point of view, the following two are the most interesting ones.

4.1. Prescribed Damping Ratio. A quite natural option is to force all modes to be damped with a prescribed minimum damping ratio $\zeta_{\min } \in[0,1]$. From (19), one can see that if $k$ is fixed then with increasing value of $b$, the least damped mode is that corresponding to $\lambda_{\min }$. The ratio of imaginary and real part of the least damped mode that corresponds to $\lambda_{\min }$ is given by

$$
\xi_{\max }=\left|\frac{\operatorname{Im}\left(s_{1}\right)}{\operatorname{Re}\left(s_{1}\right)}\right|=\frac{\sqrt{4 \lambda_{\min }\left(k_{0}+k\right)-\lambda_{\min }^{2}\left(b_{0}+b\right)^{2}}}{\lambda_{\min }\left(b_{0}+b\right)} .
$$

Hence, the minimum value of $b$ satisfying this condition is given by

$$
b_{\mathrm{damp}}=\sqrt{\frac{4\left(k_{0}+k_{\mathrm{damp}}\right)}{\lambda_{\min }\left(\xi_{\max }^{2}+1\right)}}-b_{0}
$$

where $k_{\text {damp }}$ is set arbitrarily.

Since the corresponding damping ratio is given as

$$
\zeta_{\min }=\sqrt{\frac{1}{1+\xi_{\max }^{2}}},
$$

after substitution into (21), we obtain

$$
b_{\text {damp }}=2 \zeta_{\min } \sqrt{\frac{k_{0}+k_{\text {damp }}}{\lambda_{\text {min }}}}-b_{0} .
$$

Let us define stability margin as

$$
\delta=\min _{\ell}\left|\operatorname{Re}\left(s_{\ell}\right)\right| \text {. }
$$

Stability margin of such a control law is determined by the distance of the least and most damped closed-loop eigenvalues, $s_{1}$ and $s_{n}^{+}$, respectively, from the imaginary axis 


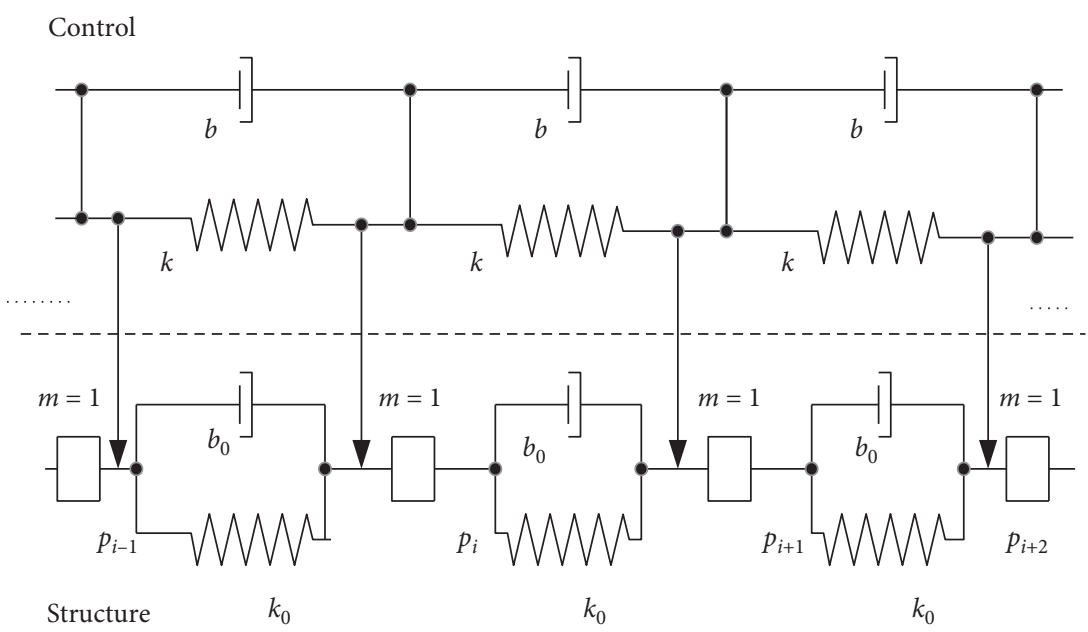

Figure 1: Distributed control law of a one-dimensional structure.

for $b=b_{\text {damp }}, k=k_{\text {damp. }}$. The distance of complex conjugate $s_{1}^{ \pm}$corresponding to $\lambda_{\min }$ whose position is given by the prescribed damping ratio is given by

$$
d\left(s_{1}^{ \pm}\right)=-\operatorname{Re}\left(s_{1}\right)=\frac{\lambda_{\min }\left(b_{0}+b_{\text {damp }}\right)}{2},
$$

whereas distance of real $s_{n}^{+}$corresponding to $\lambda_{\max }$ can be obtained as

$$
d\left(s_{n}^{+}\right)=\frac{\lambda_{\max }\left(b_{0}+b_{\text {damp }}\right)-\sqrt{\lambda_{\max }^{2}\left(b_{0}+b_{\text {damp }}\right)^{2}-4 \lambda_{\max }\left(k_{0}+k_{\text {damp }}\right)}}{2} .
$$
(26):

Stability margin is then given as minimum of (25) and

$$
d_{\mathrm{damp}}=\min \left\{d\left(s_{1}^{ \pm}\right), d\left(s_{n}^{+}\right)\right\},
$$

which after some algebraic manipulations yields

$$
\begin{aligned}
d_{\mathrm{damp}}= & \min \left\{\zeta_{\min } \lambda_{\min } \sqrt{k_{0}+k_{\mathrm{damp}}}, \sqrt{\lambda_{\max }\left(k_{0}+k_{\mathrm{damp}}\right)}\right. \\
& \left.\left(\zeta_{\min } \sqrt{\frac{\lambda_{\text {max }}}{\lambda_{\min }}}-\sqrt{\zeta_{\min }^{2} \frac{\lambda_{\max }}{\lambda_{\min }}-1}\right)\right\} .
\end{aligned}
$$

It should be noted that, for very small damping $\left(\zeta_{\min }<\lambda_{\min } / \lambda_{\max }\right)$, the eigenvalue $s_{n}$ is not real and the second term in (28) becomes complex and should not be considered. Nevertheless, considering such a small damping is highly impractical.

The dependence of stability margin on prescribed minimum damping ratio given by (28) for $n=48$ and $k_{0}+$ $k_{\text {damp }}=1$ is depicted in Figure 2.

4.2. Maximum Stability Margin. Another interesting option is to find the control parameters that maximize the stability margin (24). From (19), it follows that the stability margin of the eigenvalues lying on real axis is determined by that

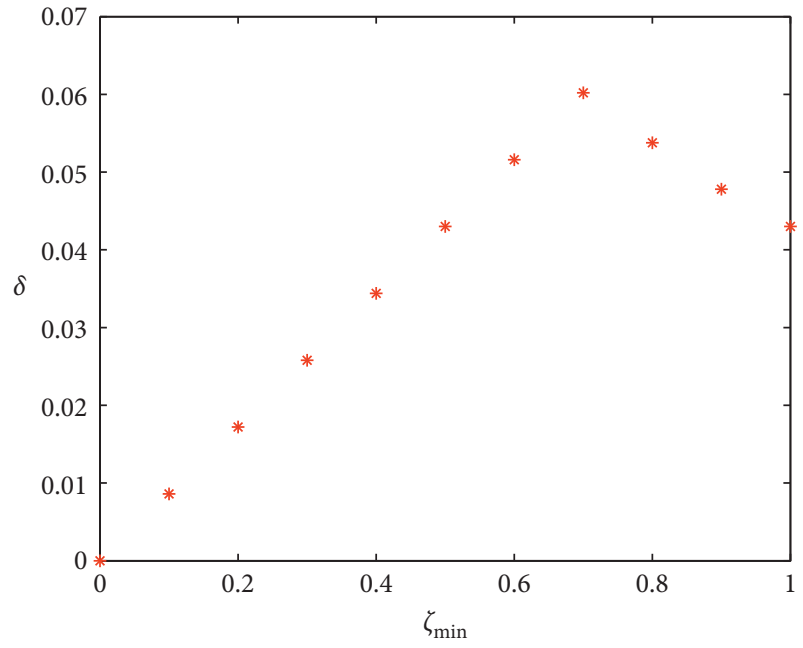

FIgURE 2: Stability margin dependence on damping ratio.

corresponding to $\lambda_{\max }$ and stability margin of the eigenvalues lying out of real axis is determined by those corresponding to $\lambda_{\min }$. Thus, the maximum stability margin is achieved if

$$
\operatorname{Re}\left(s_{1}^{ \pm}\right)=\operatorname{Re}\left(s_{n}^{+}\right),
$$

where $s_{1}$ and $s_{n}$ are the eigenvalues corresponding to $\lambda_{\text {min }}$ and $\lambda_{\max }$, respectively.

Condition (29) can be written as

$$
-\lambda_{\min }\left(b_{0}+b\right)=-\lambda_{\max }\left(b_{0}+b\right)+\sqrt{\lambda_{\max }^{2}\left(b_{0}+b\right)^{2}-4 \lambda_{\max }\left(k_{0}+k\right)},
$$

from which we obtain

$$
b_{\text {marg }}=\sqrt{\frac{4 \lambda_{\max }\left(k_{0}+k_{\operatorname{marg}}\right)}{\lambda_{\min }\left(2 \lambda_{\max }-\lambda_{\min }\right)}}-b_{0},
$$

for arbitrarily chosen $k_{\text {marg }}$. 
By substituting (31) in (29), we obtain the maximum stability margin as

$$
\delta_{\max }=\sqrt{k_{0}+k_{\operatorname{marg}}} \sqrt{\frac{\lambda_{\max } \lambda_{\min }}{2 \lambda_{\max }-\lambda_{\min }}} .
$$

The ratio of imaginary and real part of the least damped mode that corresponds to $\lambda_{\min }$ is given by

$$
\xi_{\max }=\left|\frac{\operatorname{Im}\left(s_{1}\right)}{\operatorname{Re}\left(s_{1}\right)}\right|=\frac{\sqrt{4 \lambda_{\min }\left(k_{0}+k_{\operatorname{marg}}\right)-\lambda_{\min }^{2}\left(b_{0}+b_{\text {marg }}\right)^{2}}}{\lambda_{\min }\left(b_{0}+b_{\text {marg }}\right)},
$$

that yields after substitution from (31) and some simplifications

$$
\xi_{\max }=\sqrt{1-\frac{\lambda_{\min }}{\lambda_{\max }}}
$$

From that, the damping ratio of the least damping mode follows as

$$
\zeta_{\min }=\sqrt{\frac{1}{1+\xi_{\max }^{2}}}=\sqrt{\frac{\lambda_{\max }}{2 \lambda_{\max }-\lambda_{\min }}} .
$$

The achievable stability margins for different values of $n$ for both approaches are shown in Figure 3, whereas the minimum damping ratio corresponding to maximum stability margin is depicted in Figure 4. Both figures are plotted for $k_{0}+k_{\text {marg }}=1$.

\section{Example}

Let us illustrate the results derived in the previous section on an example. We will consider the following parameters: $m=5 \cdot 10^{-4} \mathrm{~kg}, \bar{b}=3.3 \cdot 10^{-3} \mathrm{Ns} / \mathrm{m} ; \bar{k}=0.4 \mathrm{~N} / \mathrm{m}$, and $n=48$. We set the control parameter $k=k_{\text {damp }}=k_{\text {marg }}$ $=10 \mathrm{~N} / \mathrm{m}$.

The minimum and maximum eigenvalues of the grounded Laplacian become

$$
\begin{aligned}
& \lambda_{\text {min }}=2-2 \cos \left(\frac{\pi}{n+1}\right)=0.0041, \\
& \lambda_{\text {max }}=2-2 \cos \left(\frac{n \pi}{n+1}\right)=3.99 .
\end{aligned}
$$

The minimum value of $b=b_{\text {damp }}$ damping all the eigenvalues with minimum damping ratio $\zeta_{\min }=0.6$ is given by

$$
b_{\text {damp }}=2 \zeta_{\min } \sqrt{\frac{k_{0}+k_{\text {damp }}}{\lambda_{\min }}}-b_{0}=527 \mathrm{~s}^{-1} .
$$

Such a control law guarantees the stability margin:

$$
\delta_{\text {damp }}=\min \{1.521,1.095\}=1.095 \text {. }
$$

The position of dominant open- and closed-loop eigenvalues is plotted in Figure 5.

The value of control parameter $b$ guaranteeing maximum stability margin yields

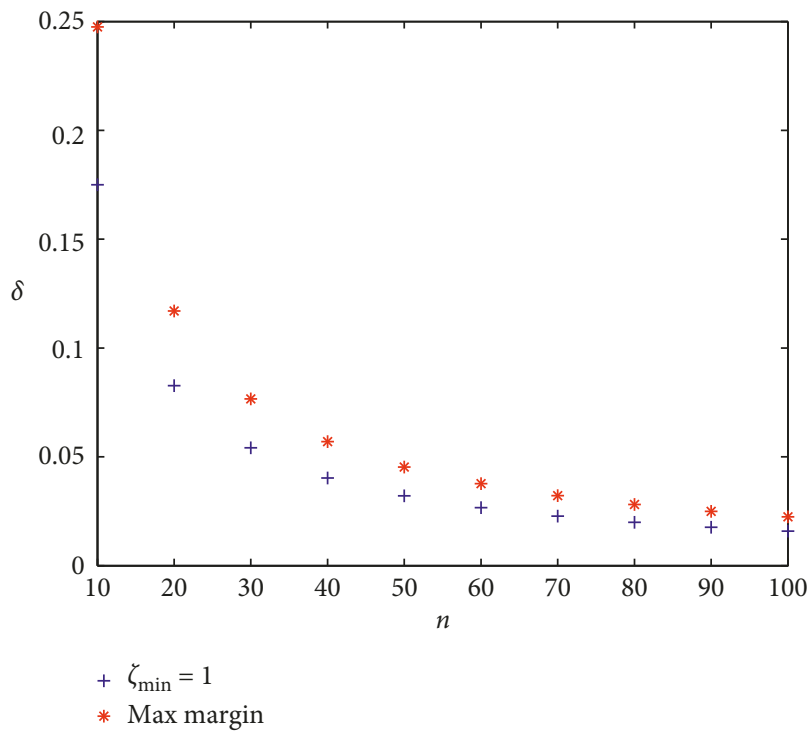

FIGURE 3: Comparison of stability margins of both approaches depending on number of nodes.

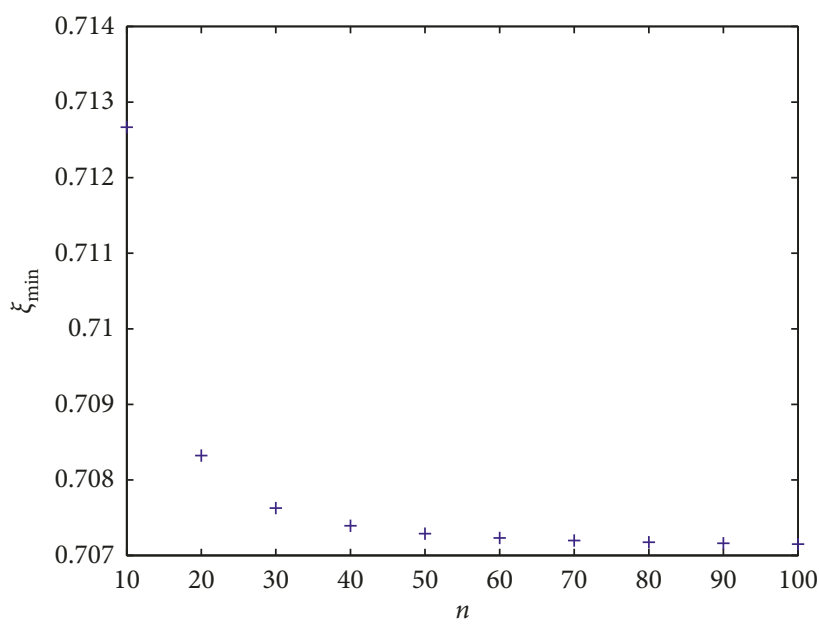

FIgURE 4: Minimum damping ratio for maximum stability margin approach depending on number of nodes.

$$
b_{\text {marg }}=\sqrt{\frac{4 \lambda_{\max }\left(k_{0}+k_{\operatorname{marg}}\right)}{\lambda_{\min }\left(2 \lambda_{\max }-\lambda_{\min }\right)}}-b_{0}=622 \mathrm{~s}^{-1},
$$

corresponding to stability margin:

$$
\delta_{\max }=\sqrt{k_{0}+k_{\operatorname{marg}}} \sqrt{\frac{\lambda_{\max } \lambda_{\min }}{2 \lambda_{\max }-\lambda_{\min }}}=1.29 .
$$

Damping ratio of the least damped mode is given by

$$
\zeta_{\min }=\sqrt{\frac{\lambda_{\max }}{2 \lambda_{\max }-\lambda_{\min }}}=0.707 .
$$

The corresponding position of dominant open- and closed-loop eigenvalues is depicted in Figure 6. 


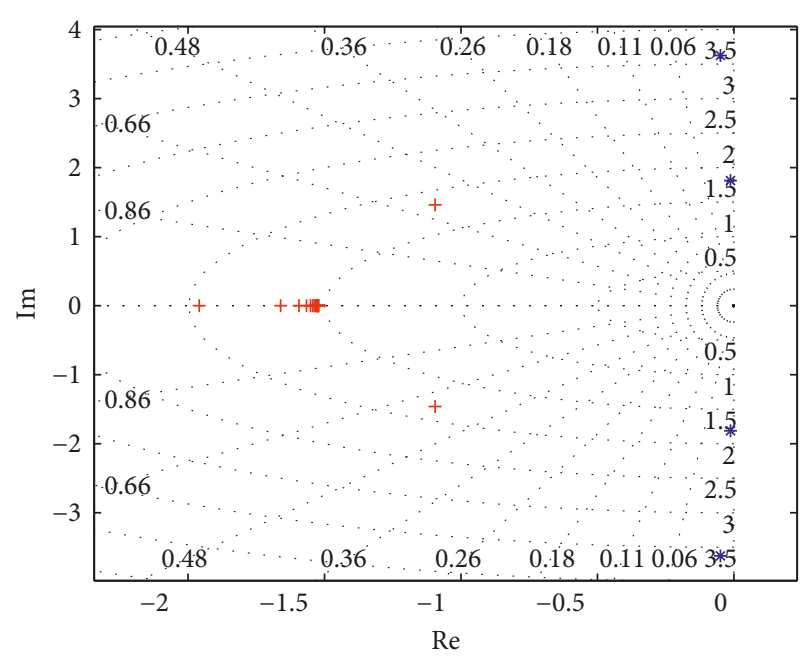

* Open-loop eig

+ Closed-loop eig

Figure 5: Dominant modes for minimum damping ratio $\zeta_{\min }=0.6$.

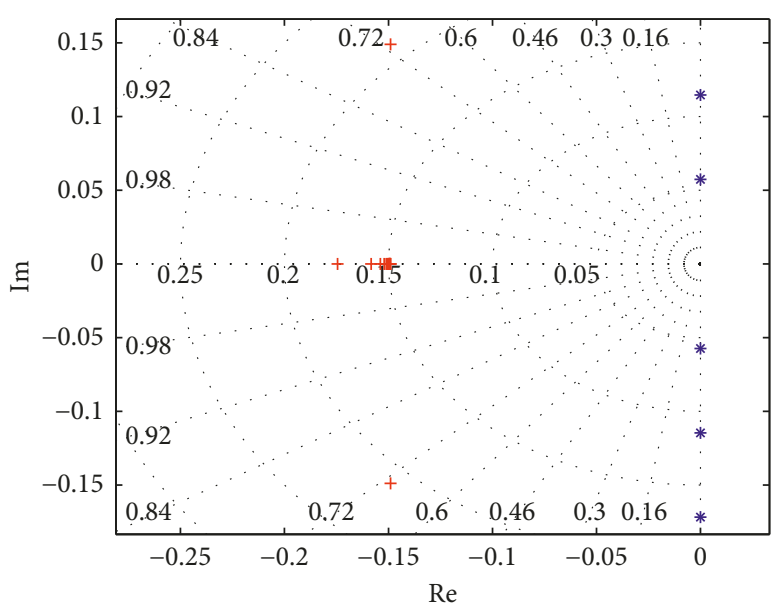

* Open-loop eig

+ Closed-loop eig

FIgURE 6: Dominant modes for maximum stability margin approach.

To demonstrate the presented design, we compare time and frequency responses of a point lying in the middle of the beam for different values of damping ratios. The open-loop responses to initial condition $p_{i}(0)=0.01 \mathrm{~m}, \dot{p}_{i}(0)=0, i=$ $1, \ldots, n$ are shown in Figure 7.

The initial condition response for different prescribed minimum damping ratios is depicted in Figure 8. The Bode plots are compared in Figure 9.

Let us compare the achieved results with other two standard design methods typically used by the control community. At first, we design an LQ controller with relative positions and velocities considered as measurable state variables, i.e.,

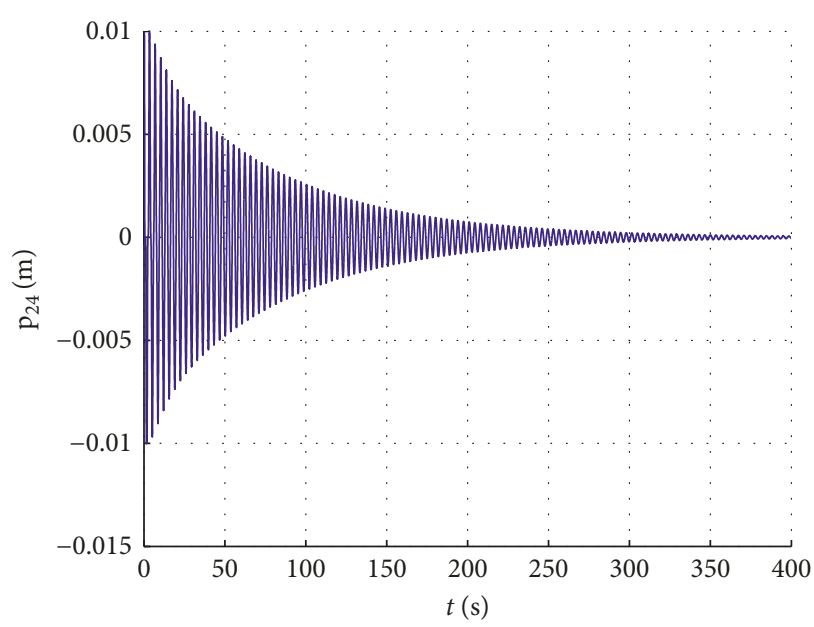

FIGURE 7: Initial condition displacement response of uncontrolled structure.

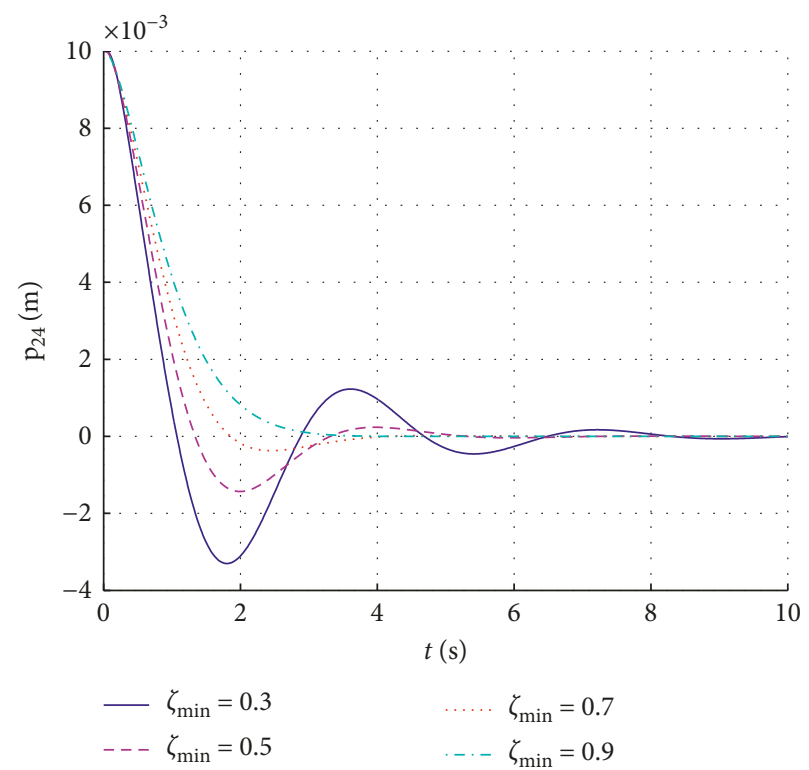

FIGURE 8: Initial condition displacement response for different damping ratios.

$$
\begin{gathered}
u_{i}=\sum_{j=1}^{n}\left(-k_{i j}\left(p_{j+1}-p_{j}\right)-k_{i j}\left(p_{j+1}-p_{j+2}\right)-b_{i j}\left(\dot{p}_{j+1}-\dot{p}_{j}\right)\right. \\
\left.-b_{i j}\left(\dot{p}_{j+1}-\dot{p}_{j+2}\right)\right), \quad i=1, \ldots, n .
\end{gathered}
$$

The difference between the LQ and presented control law is that the LQ control law uses relative positions and velocities between all neighboring nodes and not between the closest neighbors only as in (11). To force the LQ control to use relative positions and velocities, we introduce a modified state vector: 


$$
\begin{aligned}
z= & {\left[p_{2}-p_{1}+p_{2}-p_{3}, \dot{p}_{2}-\dot{p}_{1}+\dot{p}_{2}-\dot{p}_{3}, \ldots, p_{n+1}\right.} \\
& \left.-p_{n}+p_{n+1}-p_{n+2}, \dot{p}_{n+1}-\dot{p}_{n}+\dot{p}_{n+1}-\dot{p}_{n+2}\right]^{\mathrm{T}} .
\end{aligned}
$$

The criterion to be minimized is then given by

$$
J=\int_{0}^{\infty}\left(z^{\mathrm{T}} \mathrm{Q} z+u^{\mathrm{T}} R u\right) d t,
$$

resulting in LQ feedback control law

$$
u=K z \text {, }
$$

with

$$
K=\left[\begin{array}{ccccccc}
k_{22} & b_{22} & k_{23} & b_{23} & \cdots & k_{2 n+1} & b_{2 n+1} \\
k_{32} & b_{32} & k_{33} & b_{33} & \cdots & k_{3 n+1} & b_{3 n+1} \\
\vdots & \vdots & \vdots & \vdots & \cdots & \vdots & \vdots \\
k_{n+12} & b_{n+12} & k_{n+13} & b_{n+13} & \cdots & k_{n+1 n+1} & b_{n+1 n+1}
\end{array}\right] .
$$

By tuning the weighting matrices $Q$ and $R$ to guarantee minimum damping ratio $\zeta_{\min }=0.6$ we obtained the corresponding stability margin $\delta_{\text {damp }}=1.162$, see dominant poles in Figure 10. Tuning the weighting matrices to maximize stability margin, we arrived to $\delta_{\max }=1.01$ with corresponding $\zeta_{\min }=0.903$ that can be seen from dominant poles in Figure 11. Hence, the dominant poles configuration is very similar to the proposed design (Figures 5 and 6).

The control gains $k_{i j}$ and $b_{i j}$ for the former case are depicted in Figures 12 and 13, respectively. One can see that the control law uses the relative positions and velocities to the closest neighbors only and that the gains are almost the same for all nodes.

To compare our methodology with another control design approach, we formulated the task as an $H_{\infty}$ design for the fixed structure controller (11). It can be easily done with hinfstruct() function in Matlab Robust Control Toolbox. This tuning minimizes the $H_{\infty}$ norm of the closed-loop transfer function modeled by the closed-loop control system with tunable components and weighting filters. In our case, the high-pass filter with cutoff frequency $8 \mathrm{rad} / \mathrm{s}$ has been used to penalize all system modes.

The $H$-infinity design methodology offers efficient algorithms how to obtain multivariable control laws by specifying closed-loop frequency response requirements. This approach was used, for e.g., in [33], where authors compare classical single-input single-output controllers with $H$-infinity approaches in terms of robustness and performance. The order of the $H$-infinity control system is however equal to the so-called augmented plant containing the model of the controlled system along with the weighting filters defining performance and robustness requirements. This leads to excessively high-order control laws typically, with strong negative impact on implementation and experimental fine-tuning. For this reason, e.g., in [34], there was a method presented for the controller order reduction which is one possible way how to get control laws with reasonable complexity. Nevertheless, loss or deterioration

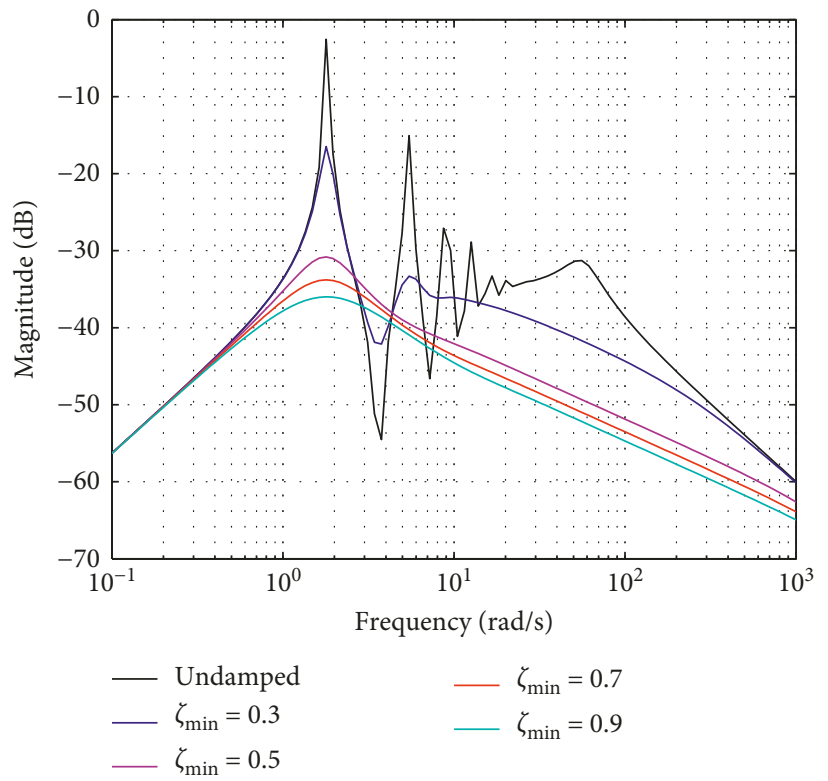

FIGURE 9: Bode plot for different prescribed minimum damping ratios.

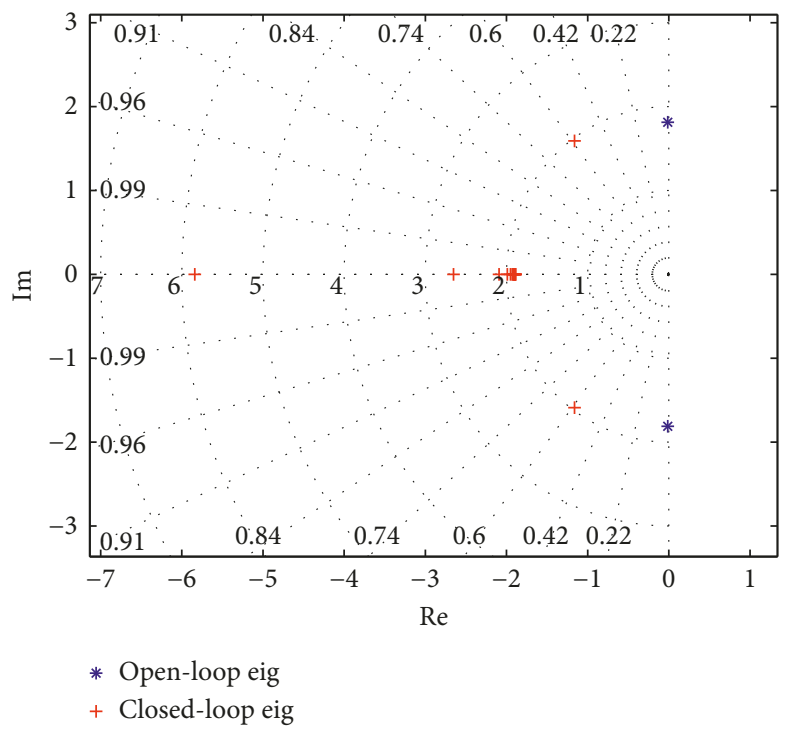

FIgUre 10: Dominant poles for LQ controller design, $\zeta_{\min }=0.6$.

of closed-loop performance and/or stability is often an unwanted effect associated with this approach. Thanks to recent structured H-infinity control synthesis results (see, e.g., $[35,36])$, it is possible to receive the parameters of such reduced-order controllers directly, minimizing the $\mathrm{H}$-infinity norm under the controller complexity constraints.

The Bode plots of original system, LQ controller tuned for prescribed minimum damping ratio $\zeta_{\min }=0.6$, the $H_{\infty}$ controller, and the proposed design for $\zeta_{\min }=0.6$ are compared in Figure 14. The plots confirm that all designs give very similar results.

\section{Conclusion}

In the paper, we presented an active approach of onedimensional structures with dense array of collocated 


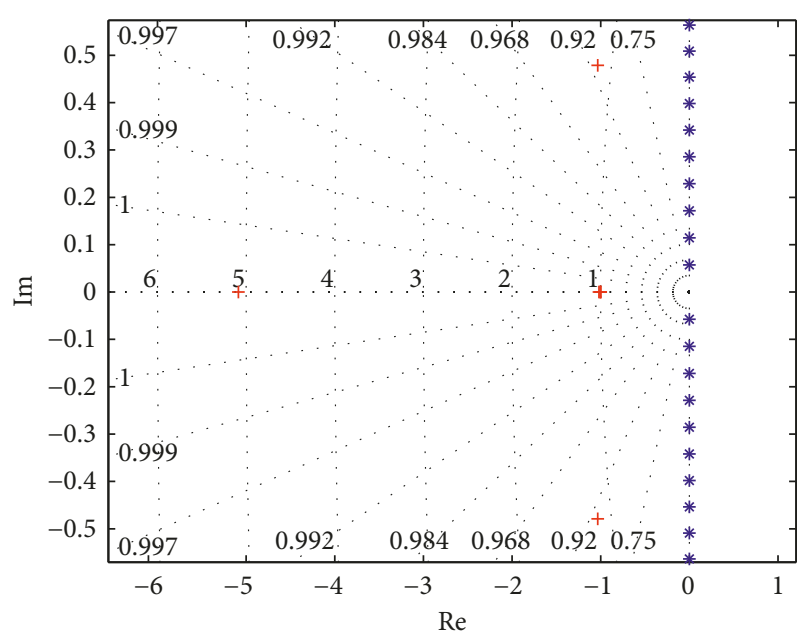

* Open-loop eig

+ Closed-loop eig

FIgURE 11: Dominant poles for LQ controller design-maximum stability margin.

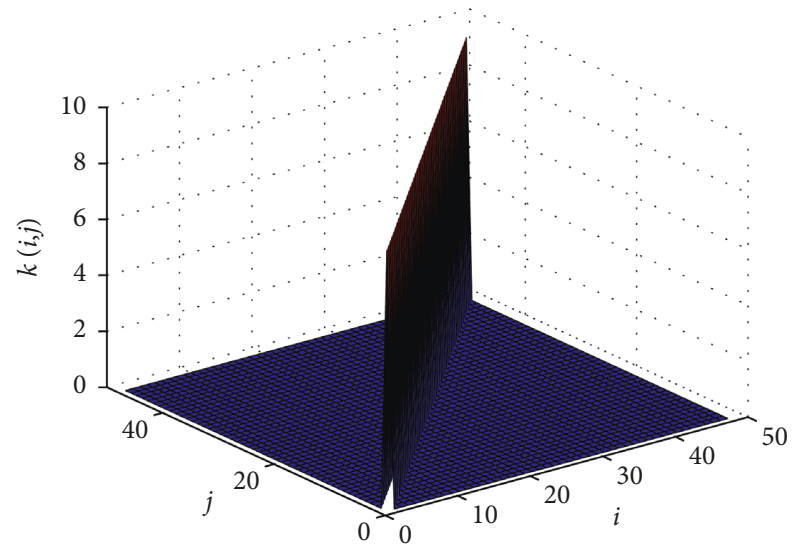

FIGURE 12: Control gains $k_{i j}$ for LQ controller design, $\zeta=0.6$.

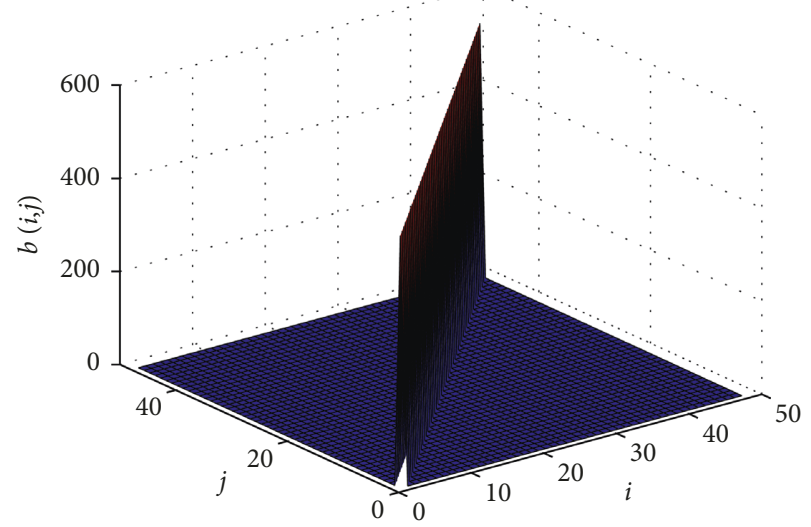

FIgure 13: Control gains $b_{i j}$ for LQ controller design, $\zeta=0.6$.

sensors and actuators using proportional position and velocity feedback control laws. The control law was formulated in a distributive manner, i.e., each actuator uses information from its closest neighbors only. The achievable stability margins and

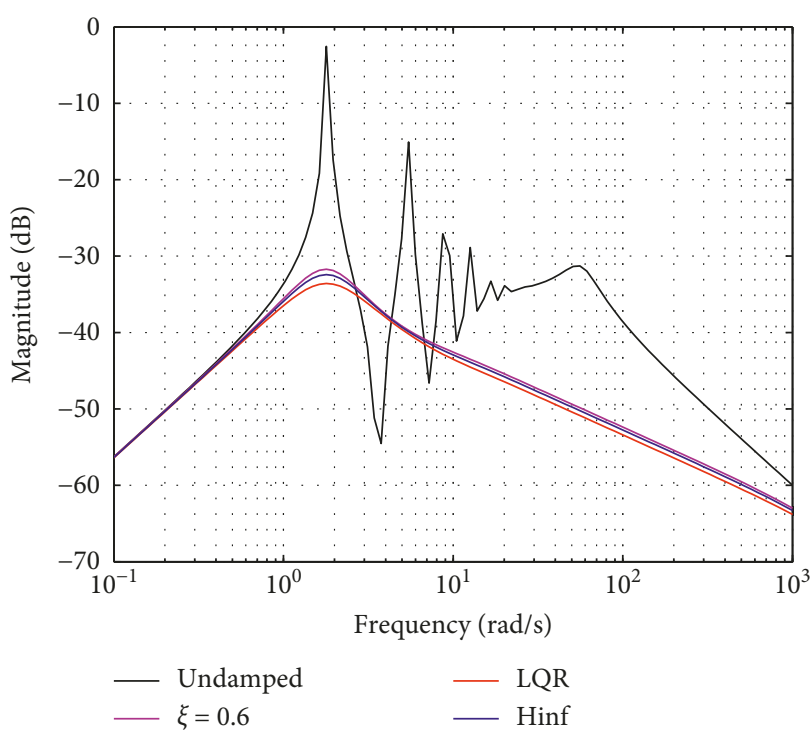

Figure 14: Bode plot for displacement for different control strategies.

damping ratios were analyzed based on the properties of Laplacian matrix describing the corresponding information graph. Comparison with LQ controller and $H_{\infty}$ designs shows that the presented approach achieves similar results yet with much lower computational and actuator complexity.

\section{Data Availability}

The data used to support the findings of this study are available from the corresponding author upon request.

\section{Conflicts of Interest}

The authors declare that they have no conflicts of interest.

\section{Acknowledgments}

The presented research has been supported by the Czech Science Foundation under the project No. 16-21961S.

\section{References}

[1] M. Kozek and A. Schirrer, Modeling and Control for a Blended Wing Body Aircraft, Springer, Berlin, Germany, 2015.

[2] R. R. Craig, "Coupling of substructures for dynamic analyses: an overview," in Proceedings of 41st Structures, Structural Dynamics, and Materials Conference and Exhibit, pp. 15731584, Atlanta, Georgia, April 2000.

[3] P. Verboven, Frequency-domain system identification for modal analysis, Ph.D. thesis, Mechanical Engineering Department (WERK), Vrije Universiteit Brussel, Brussels, Belgium, 2002.

[4] W. K. Gawronski, Advanced Structural Dynamics and Active Control of Structures, Springer-Verlag New York, Inc., New York, NY, USA, 2004.

[5] T. Hanis and M. Hromcik, "Optimal sensors placement and spillover suppression," Mechanical Systems and Signal Processing, vol. 28, pp. 367-378, 2012. 
[6] D. C. Kammer and M. L. Tinker, "Optimal placement of triaxial accelerometers for modal vibration tests," Mechanical Systems and Signal Processing, vol. 18, no. 1, pp. 29-41, 2004.

[7] W. Liu, Z. Hou, and M. A. Demetriou, "A computational scheme for the optimal sensor/actuator placement of flexible structures using spatial $\mathrm{H}_{2}$ measures," Mechanical Systems and Signal Processing, vol. 20, no. 4, pp. 881-895, 2006.

[8] M. Alam, M. Hromcik, and T. Hanis, "Active gust load alleviation system for flexible aircraft: mixed feedforward/ feedback approach," Aerospace Science and Technology, vol. 41, pp. 122-133, 2015.

[9] J. K. Hedrick, M. Tomizuka, and P. Varaiya, "Control issues in automated highway systems," IEEE Control Systems, vol. 14, no. 6, pp. 21-32, 1994.

[10] S. Darbha and P. R. Pagilla, "Limitations of employing undirected information flow graphs for the maintenance of rigid formations for heterogeneous vehicles," International Journal of Engineering Science, vol. 48, no. 11, pp. 1164-1178, 2010.

[11] W. W. Zhang, "Root locus approach to a distributed parameter vibrating system with both bending and torsion motions," International Journal of Mechanical Sciences, vol. 37, no. 6, pp. 585-600, 1995.

[12] M. Krommer, H. Irschik, and M. Zellhofer, "Design of actuator networks for dynamic displacement tracking of beams," Mechanics of Advanced Materials and Structures, vol. 15, no. 3-4, pp. 235-249, 2008.

[13] H. Irschik and M. Nader, "Actuator placement in static bending of smart beams utilizing Mohr's analogy," Engineering Structures, vol. 31, no. 8, pp. 1698-1706, 2009.

[14] E. Omidi, S. N. Mahmoodi, and W. S. Shepard, "Multi positive feedback control method for active vibration suppression in flexible structures," Mechatronics, vol. 33, pp. 23-33, 2016.

[15] L. Gaul and J. Becker, "Reduction of structural vibrations by passive and semiactively controlled friction dampers," Shock and Vibration, vol. 2014, Article ID 870564, 7 pages, 2014.

[16] D. Hughes and J. T. Wen, "Passivity motivated controller design for flexible structures," Journal of Guidance, Control, and Dynamics, vol. 19, no. 3, pp. 726-729, 1996.

[17] D. Halim and S. O. R. Moheimani, "Spatial resonant control of flexible structures-application to a piezoelectric laminate beam," IEEE Transactions on Control Systems Technology, vol. 9, no. 1, pp. 37-53, 2001.

[18] T. Komatsuzaki, T. Inoue, and O. Terashima, "Broadband vibration control of a structure by using a magnetorheological elastomer-based tuned dynamic absorber," Mechatronics, vol. 40, pp. 128-136, 2016.

[19] J. Dayou and S. M. Kim, "Control of kinetic energy of a onedimensional structure using multiple vibration neutralizers," Journal of Sound and Vibration, vol. 281, no. 1-2, pp. 323-340, 2005.

[20] J. Dayou, "Fixed-points theory for global vibration control using vibration neutralizer," Journal of Sound and Vibration, vol. 262, no. 3-5, pp. 765-776, 2006.

[21] R. Chen and T. Wu, "Vibration control of base system using distributed dynamic vibration absorbers," Journal of Vibration and Control, vol. 20, no. 10, pp. 1589-1600, 2014.

[22] S. S. Patil and P. J. Awasare, "Vibration reduction at desired locations on a beam by creating nodes using tunable vibration neutralizers," Journal of Vibration and Control, vol. 22, no. 1, pp. 205-223, 2016.

[23] M. Azoulay, A. Veprik, V. Babitsky, and N. Halliwell, "Distributed absorber for noise and vibration control," Shock and Vibration, vol. 18, no. 1-2, pp. 181-219, 2011.
[24] G. Takacs, G. Batista, M. Gulan, and B. Rohal-Ilkiv, "Embedded explicit model predictive vibration control," Mechatronics, vol. 36, pp. 54-62, 2017.

[25] P. Gardonio and S. J. Elliott, "Active control of waves on a one-dimensional structure with a scattering termination," Journal of Sound and Vibration, vol. 192, no. 3, pp. 701-730, 1996.

[26] R. Gabai and I. Bucher, "Excitation and sensing of multiple vibrating traveling waves in one-dimensional structures," Journal of Sound and Vibration, vol. 319, no. 1-2, pp. 406-425, 2009.

[27] F. Li, C. Zhang, and C. Liu, "Active tuning of vibration and wave propagation in elastic beams with periodically placed piezoelectric actuator/sensor pairs," Journal of Sound and Vibration, vol. 393, pp. 14-29, 2017.

[28] S. Chen, G. Wang, J. Wen, and X. Wen, "Wave propagation and attenuation in plates with periodic arrays of shunted piezo-patches," Journal of Sound and Vibration, vol. 332, no. 6, pp. 1520-1532, 2013.

[29] D. Ning, S. Sun, L. Wei, B. Zhang, and W. Li, "Vibration reduction of seat suspension using observer based terminal sliding mode control with acceleration data fusion," Mechatronics, vol. 44, pp. 71-83, 2017.

[30] Y. Zhang, B. Kosmatopoulos, P. A. Ioannou, and C. C. Chien, "Using front and back information for tight vehicle following maneuvers," IEEE Transactions on Vehicular Technology, vol. 48, no. 1, pp. 319-328, 1999.

[31] P. Barooah, P. G. Mehta, and J. P. Hespanha, "Mistuningbased decentralized control of vehicular platoons for improved closed loop stability," IEEE Transactions on Automatic Control, vol. 54, no. 9, pp. 2100-2113, 2009.

[32] H. Hao and P. Barooah, "On achieving size-independent stability margin of vehicular lattice formations with distributed control," IEEE Transactions on Automatic Control, vol. 57, no. 10, pp. 2688-2694, 2012.

[33] M. R. Waszak, "Robust multivariable flutter suppression for banchmark active control technology wind-tunnel model," Journal of Guidance, Control, and Dynamics, vol. 24, no. 1, pp. 147-153, 2001.

[34] A. Varga, "Balancing free square-root algorithm for computing singular perturbation approximations," in Proceedings of 30th Conference on Decision and Control, Brighton, UK, December 1991.

[35] P. Apkarian and D. Noll, "Nonsmooth Ho Synthesis," IEEE Transactions on Automatic Control, vol. 51, no. 1, pp. 71-86, 2006.

[36] S. Gumussoy, D. Henrion, M. Millstone, and M. L. Overton, "Multiobjective robust control with HIFOO 2.0," in Proceedings of the IFAC Symposium on Robust Control Design, pp. 144-149, Haifa, Israel, June 2009. 


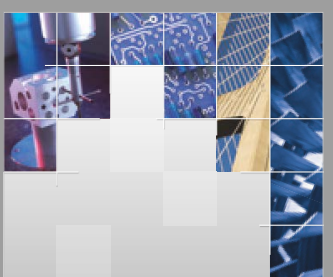

\section{Enfincering}
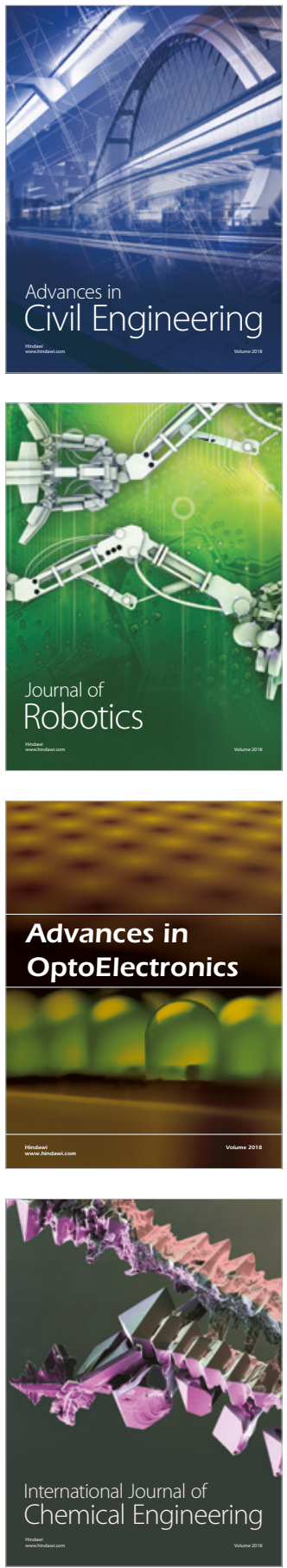

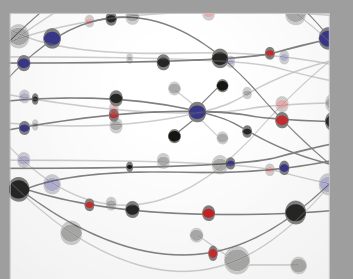

\section{Rotating \\ Machinery}

The Scientific World Journal

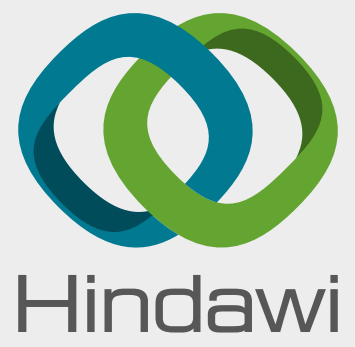

Submit your manuscripts at

www.hindawi.com
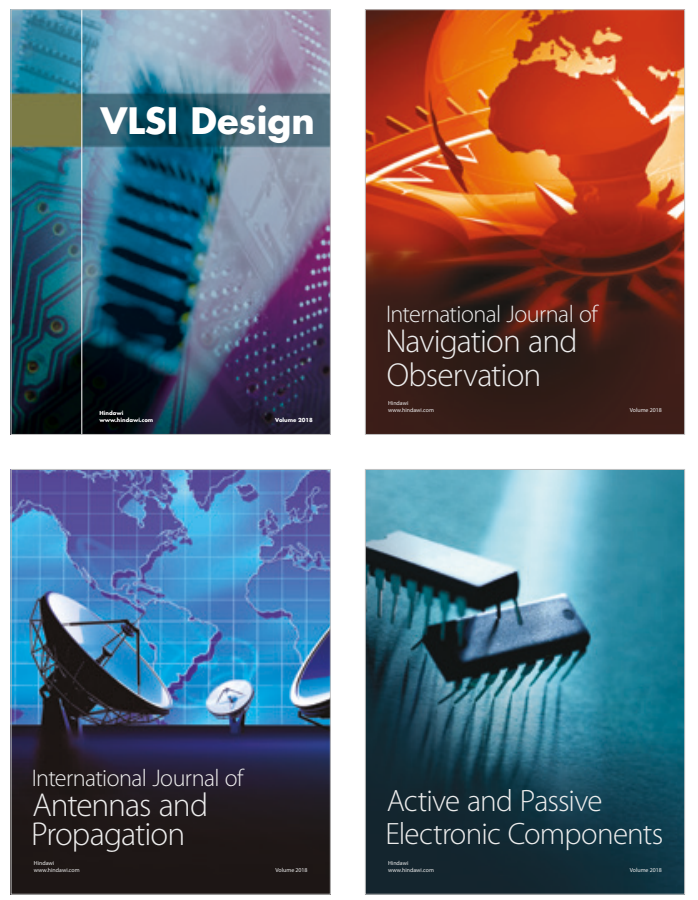
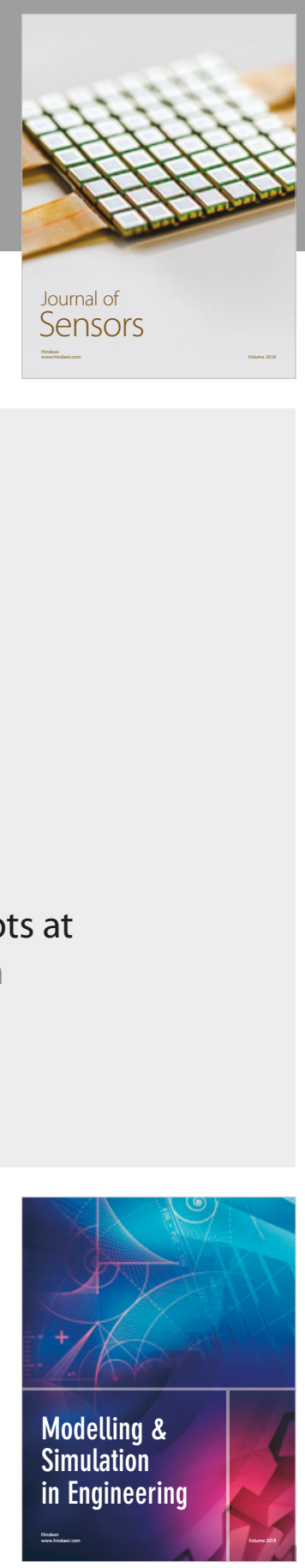

\section{Advances \\ Multimedia}
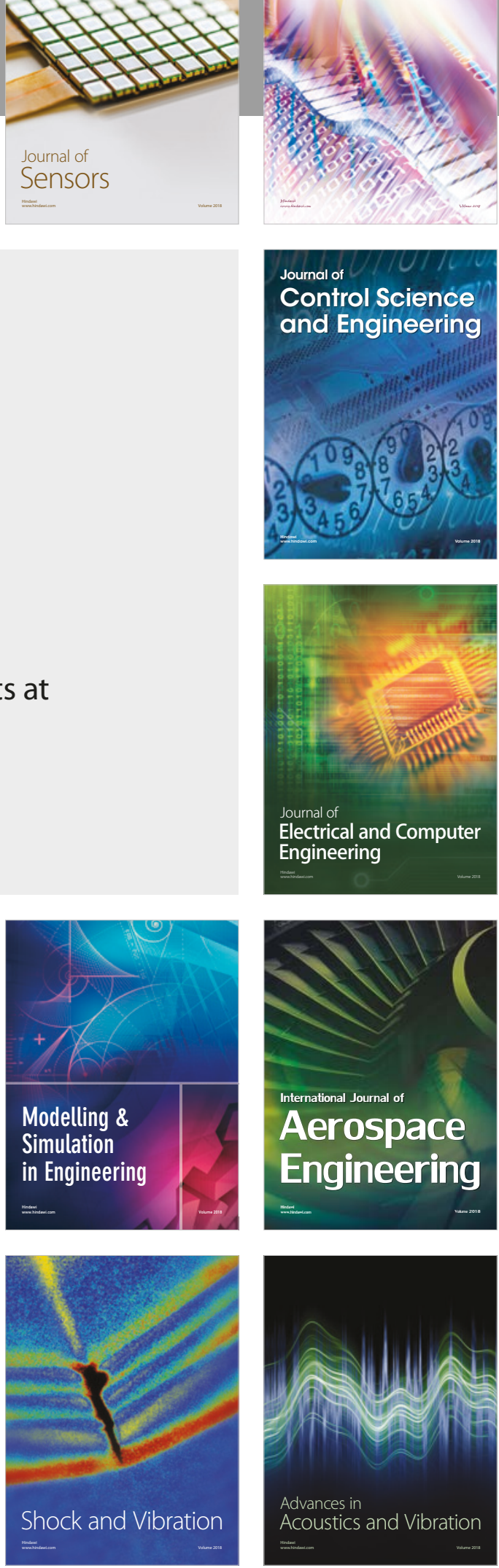